\title{
IL-32 and IL-17 interact and have the potential to aggravate osteoclastogenesis in rheumatoid arthritis
}

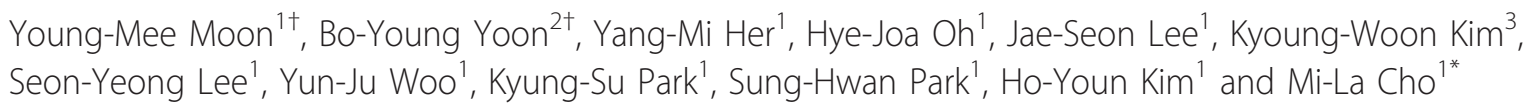

\begin{abstract}
Introduction: Interleukin (IL)-32 and IL-17 play critical roles in pro-inflammatory responses and are highly expressed in the synovium of patients with rheumatoid arthritis (RA). We investigated the relations between these two cytokines (IL-17 and IL-32) for their ability to induce each other and to stimulate osteoclasts in RA fibroblastlike synoviocytes (FLSs) and T cells.

Methods: FLSs were isolated through surgical synovectomy obtained from patients with RA or osteoarthritis (OA). Real-time PCR were performed to evaluate the expression of IL-32, IL-17 and osteoclast-related genes. Immunohistochemical staining and tartrate-resistant acid phosphatase (TRAP) staining were performed to determine the distribution of inflammatory cytokines and the presence of osteoclastogenesis.

Results: IL-17 induced the expression of IL-32 in the FLSs from RA patients, as assessed by microarray. IL-32 production was increased by IL-17. IL-32 in the FLSs from RA patients induced the production of IL-17 in CD4 T cells. IL-32 and IL-17 were colocalized near TRAP-positive areas in joint specimens. IL-17 and IL-32 synergistically induced the differentiation of osteoclasts, as demonstrated by the expression of osteoclast-related genes. IL-32 and IL-17 also could induce resorption by osteoclasts in a RANKL-dependent manner.

Conclusions: IL-17 affected the expression of IL-32 in FLSs of RA patients and IL-32 induced the production of IL-17 in CD4+ T cells. Both IL-17 and IL-32 cytokines can reciprocally influence each other's production and amplify the function of osteoclastogenesis in the in RA synovium. Separately, IL-17 and IL-32 each stimulated osteoclastogenesis without RANKL. Together, the two cytokines synergistically amplified the differentiation of osteoclasts, independent of RANKL stimulation.
\end{abstract}

\section{Introduction}

Rheumatoid arthritis (RA) is a chronic systemic autoimmune disease that predominantly affects multiple peripheral synovial joints. The synovial environment has numerous inflammatory cells such as T cells, B cells, fibroblast-like synoviocytes (FLSs) and antigen-presenting cells, which can cause the development of RA. FLSs constitute the synovial lining cells that have a key role in pannus formation and destruction of joints [1]. In addition, numerous cytokines

\footnotetext{
* Correspondence: iammila@catholic.ac.kr

† Contributed equally

${ }^{1}$ The Rheumatism Research Center, Catholic Research Institute of Medical Science, The Catholic University of Korea, 505 Banpo-dong, Seocho-gu, Seoul 137-701, South Korea

Full list of author information is available at the end of the article
}

have been implicated in the immune processes that are associated with RA. T cells, the most invading type of lymphocyte in the RA synovium, can contact and activate FLSs $[2,3]$. A variety of in vitro and in vivo models have shown that TNF-dependent networks are involved in critical pathogenic interactions in RA synovitis. In recent years, new novel cytokines such as IL-17 and IL-32 have been reported to be involved in the pathogenesis or regulation of synovial inflammation.

IL-17, a proinflammatory cytokine produced by T helper (Th)17 cells [4], plays a key role in the propagation of joint inflammation, cartilage destruction and bone erosion[5,6], and it is present in both the synovium and synovial fluid [7]. IL-17 participates in the joint inflammation of RA via 
activation of T cells and FLSs by secreting cytokines and chemokines such as IL-6, IL-8, 1L-16, stromal cell-derived factor-1 (SDF-1), matrix metalloproteinase (MMP)-3 and MMP-1 [8-11]. Moreover, IL-17 is well known as a strong inducer of osteoclastogenesis [5].

IL-32, a recently discovered cytokine, was originally described as natural killer (NK) cell transcript 4 (NK4) [12]. IL-32 has four splice variants, $I L-32 \alpha, I L-32 \beta, I L-32 \delta$ and $I L-32 \gamma$, with $I L-32 \alpha$ as the most abundant transcript, and $I L-32 \gamma$ as the most active isoform. The expression of TNF- $\alpha$ and IL- 6 are significantly correlated with IL-32 $\gamma$ expression [13]. IL-32 is expressed in NK cells, T cells, epithelial cells and blood monocytes upon stimulation by inflammatory cytokines such as IL- $1 \beta$, IL-18, IFN- $\gamma$ and TNF- $\alpha$ by the phosphatidylinositol 3-kinase/Akt and NF$\kappa \mathrm{B} / \mathrm{AP}-1$ systems [14]. IL-32 is highly expressed in the synovial tissue and FLSs of RA patients, but not in osteoarthritis patients [15]. IL-32 can also induce inflammatory cytokines and chemokines such as TNF- $\alpha$, IL- $1 \beta$, IL- 8 and IL- 6 by the activation of NF- $\kappa$ B and p38 mitogen-activated protein kinase [16]. Injection of human IL-32 into the knee joints of naïve mice results in joint swelling, infiltration and cartilage damage. For these reasons, IL-32 has been recognized as a proinflammatory cytokine, and has been implicated in inflammatory disorders such as RA and inflammatory bowel disease $[17,18]$.

IL-32 and IL-17 are thought to be associated with pathogenesis, and are frequently mentioned together as they seem to have similar roles. It was reported that CXC chemokine receptor 4 (CXCR4), lamina propria lymphocytes (LPL) and IL-32 were identified by IL-17A or IL-17F plus TNF $\alpha$ on RA synoviocytes [19]. Studies using RA FLSs and $\mathrm{CD}_{4}^{+} \mathrm{T}$ cells or dendritic cells have shown a reciprocal induction between TNF $\alpha$ and IL-32, creating a TNF $\alpha$ / IL-32/TNF $\alpha$-positive autoinflammatory loop [20]. Moreover, IL-32 production is partially dependent on TNF $\alpha$, and the treatment of RA patients with anti-TNF $\alpha$ has resulted in the reduction of IL-32 protein in synovial tissue. In a recent report, it was suggested that IL-32 $\gamma$ contributes to the maturation and activation of immature dendritic cells (DCs) and increases Th1 and Th17 response by IL-12 and IL-6 [21]. In addition, IL-17 and IL-32 have been shown to influence pathogenesis via the common protein $\mathrm{p} 300$ and DAPK-1, through the TNF-R1 dependent/independent pathway [22].

From these investigations, we hypothesized that IL-32 and IL-17 interact with each other, and function to amplify inflammatory reactions in RA. In this study, we examined the interaction between the two cytokines, and further investigated their synergistic involvement in osteoclastogenesis functions. Osteoclasts have a key role in the joint destruction of RA. It was reported that both IL-17 and IL-32 induce the generation of osteoclasts $[5,23,24]$. IL-17 functionally upregulates the receptor activator of NF- $\kappa \mathrm{B}$ (RANK) on osteoclast precursors causing increased sensitivity to RANK ligand (RANKL) signaling, leading to increased bone destruction [25]. A recent study showed that IL-32 $\gamma$ has a greater potential for generating osteoclasts compared to IL-17 in the presence of soluble RANKL [24].

Our results suggest that IL-17 and IL-32 stimulate each other's production, and both inflammatory cytokines synergistically induce osteoclastogenesis. Eventually, IL-17 and IL-32 might accelerate synovial inflammation and erode cartilage and bone by osteoclastogenesis in patients with RA.

\section{Materials and methods \\ Patients and mice}

FLS cell lines were prepared from the synovectomized tissue of RA patients who were undergoing joint replacement surgery [8]. Six- to eight-week-old male DBA/1J mice () were maintained for CIA induction. $I L-1 R$ antagonist-deficient mice (IL-1Ra-/- mice) in a BALB/c background were provided by Dr Y Iwakura (University of Tokyo, Tokyo, Japan). All the experimental procedures were examined and approved by the Animal Research Ethics Committee at the Catholic University of Korea.

\section{Cell preparation}

Peripheral blood was obtained from healthy donors using heparin-treated syringes. Peripheral blood mononuclear cells (PBMCs) were isolated by density centrifugation using Ficoll-Hypaque (Pharmacia LKB, Uppsala, Sweden). Mice splenocytes were isolated through a mesh and the red blood cells (RBCs) were lysed with $0.83 \%$ ammonium chloride. To purify the CD4 ${ }^{+} \mathrm{T}$ cells, the cell suspensions were incubated with CD4-coated magnetic beads (Miltenyi Biotec, Bergisch Gladbach, Germany) for 15 minutes at $4^{\circ} \mathrm{C}$ and the cells were isolated on magnetic-activated cell sorting (MACS) separation columns (Miltenyi Biotec). The CD4 ${ }^{+} \mathrm{T}$ cells were cultured with the stimuli: recombinant human IL-17, human IL-23, human IL-32 $\alpha$ (R\&D systems, Minneapolis, MN, USA), TGF- $\beta$ (Peprotech, Rocky Hill, NJ, USA), and membrane-bound anti-CD3 antibody (0.5 $\mu$ g; BD PharMingen, CA, USA), and the cells were pretreated with the inhibitors parthenolide $(10 \mu \mathrm{M})$, LY294002 (10 $\mu$ M) (A.G. Scientific, Inc., San Diego, CA, USA), or an anti-human IL-17 blocking antibody (R\&D systems) for $2 \mathrm{~h}$.

\section{Preparation of an autoimmune arthritis mice model}

To induce type 11 collagen-induced arthritis (CIA), $0.1 \mathrm{ml}$ of an emulsion containing $100 \mu \mathrm{g}$ bovine type II collagen (CII) and complete Freund's adjuvant (CFA; Chondrex, Redmond, WA, USA) was injected intradermally into the base of the tail as a primary immunization. Two weeks later, a booster injection of $100 \mathrm{ug}$ CII dissolved and 
emulsified 1:1 with incomplete Freund's adjuvant (DIFCO, Detroit, MI, USA) was administered to the hind leg.

\section{RNA preparation and real-time PCR}

The total RNA was extracted using TRI Reagent (MRC, Cincinnati, OH, USA) according to the manufacturer's instructions. The RNA concentrations were measured using a NanoDrop ND-1000 (Thermo Fisher Scientific, Waltham, MA, USA). Reverse transcription of $2 \mu \mathrm{g}$ of the total mRNA was conducted at $42^{\circ} \mathrm{C}$ using RevertAid ${ }^{\mathrm{TM}}$ M-MuLV Reverse Transcriptase and RNase inhibitor (Fermantas, Burlington, ON, Canada). PCR amplification of cDNA aliquots was performed by adding SYBR green mixture (Takara, Shiga, Japan) in a LightCycler (Roche Diagnostics Mannheim, Germany). The relative expression levels were calculated by normalizing the targets to the endogenously expressed housekeeping gene ( $\beta$-actin). Melting curve analysis was performed immediately after the amplification protocol under the following conditions: $0 \mathrm{~s}$ (hold time) at $95^{\circ} \mathrm{C}, 15 \mathrm{~s}$ at $65^{\circ} \mathrm{C}$ and $0 \mathrm{~s} \mathrm{(hold}$ time) at $95^{\circ} \mathrm{C}$. The temperature change rate was $20^{\circ} \mathrm{C} / \mathrm{s}$ except in the final step, in which it was $0.1^{\circ} \mathrm{C} / \mathrm{s}$. The crossing point $(\mathrm{Cp})$ was defined as the maximum of the second derivative from the fluorescence curve.

\section{Bead array gene expression analysis}

Total RNA (200 ng of total RNA) was used as a template for producing double-stranded cDNA and to perform in vitro transcription amplification using the Illumina Total Prep RNA amplification kit (Ambion, TX, USA), following the manufacturer's instructions. The biotin-labeled cRNA (750 ng) was purified and hybridized to the HumanRef- 8 BeadChip at $58^{\circ} \mathrm{C}$ for $16 \mathrm{~h}$ by following the Illumina whole-genome gene expression protocol for BeadStation. The arrays were scanned with the Illumina BeadArray Reader. Data normalization was performed using quantile normalization.

\section{Histology and Immunohistochemistry}

Immunohistochemical staining was performed on sections of the synovium. Briefly, the synovial samples, obtained from four patients with RA and one patient with OA, were fixed in $4 \%$ paraformaldehyde solution overnight at $4{ }^{\circ} \mathrm{C}$, dehydrated with alcohol, washed, embedded in paraffin and sectioned into $7-\mu \mathrm{m}$-thick slices. The sections were depleted of endogenous peroxidase activity by adding methanolic hydrogen peroxide $\left(\mathrm{H}_{2} \mathrm{O}_{2}\right)$ and blocking with normal serum for 30 minutes. The sections were then incubated overnight at $4^{\circ} \mathrm{C}$ with goat anti human IL-17, anti -IL-32 antibody (R\&D Systems), anti -human NF- $\kappa$ B p50 and 65 (Santa Cruz Biotechnology, Santa Cruz, CA, USA) and p-I $\kappa \mathrm{B}, \mathrm{p}-\mathrm{AKT}$ and AKT (Cell Signaling Technology, Danvers, MA, USA). A hind leg of each mouse was fixed with $1 \%$ formalin, decalcified in EDTA and embedded in paraffin wax. The sections were then stained with $\mathrm{H} \& \mathrm{E}$ and tartrate-resistant acid phosphatase (TRAP) stain. The tissues were incubated with the primary IL-17 antibody (Santa Cruz Biotechnology) and IL-32 antibody (Abfrontier, Seoul, South Korea) overnight at $4^{\circ} \mathrm{C}$. The samples were incubated with biotinylated anti-goat IgG and anti-rabbit IgG secondary antibodies for 20 minutes. The sections were then incubated with streptavidin-peroxidase complex (Vector Laboratories Ltd., Peterborough, $\mathrm{UK}$ ) for $1 \mathrm{~h}$ followed by incubation with 3, 3-diaminobenzidine (Dako, Glostrup, Denmark). The sections were counterstained with hematoxylin and the samples were photographed using a photomicroscope (Olympus, Tokyo, Japan).

\section{Osteoclast differentiation and activity}

After isolation from whole blood, human PBMCs were incubated for $3 \mathrm{~h}$. To remove nonadherent cells, the cultures were rinsed in medium. The adherent cells were cultured as osteoclast precursors using recombinant human macrophage-colony stimulating factor (M-CSF) in minimum essential medium alpha modification ( $\alpha$-MEM; Invitrogen, Carlsbad, CA, USA) and 10\% heat-inactivated fetal bovine serum (FBS) for the first 3 days. The osteoclast precursors were rinsed in medium and stimulated with MCSF and RANKL, or IL-17 or IL-32. All the factors were replenished every 3 days and the cultures were maintained for up to 21 days. A commercial TRAP kit (Sigma-Aldrich, St Louis, MO, USA) was used according to the manufacturer's instructions, and cells were counterstained with hematoxylin. TRAP-positive cells containing three or more nuclei were scored as osteoclasts. The TRAP-positive multinucleated cells (MNCs) were counted three times without the examiner having knowledge of the previously counted numbers of osteoclasts. To assess osteoclast activity, cell culture was performed as described above with dentine discs (IDS Inc., Boldon, UK) in 96-well plates. The cells were cultured for 21 days. At day 21 cells were removed from dentine discs using 10\% sodium hypochlorite solution rinsed in distilled water. The dentin discs were then stained with $50 \%$ hematoxylin rinsed in distilled water. Resorption area was evaluated by light microscopy and measured using the TMOMRO analysis Ts Lite Image program (Olympus, Munster, Germany).

\section{Enzyme-linked immunosorbent assay (ELISA)}

Antibodies for mouse IL-17 and human IL-17 were obtained from R\&D Systems. The IL-17 concentration in the culture supernatants was measured by sandwich ELISA, according to the manufacturer's instructions. A standard curve was drawn by plotting the optical density versus the log of the concentration of IL-17. 


\section{Flow cytometry analysis}

Cells were stimulated for $4 \mathrm{~h}$ with phorbol 12-myristate 13-acetate and ionomycin. The cells were permeabilized using a cytoperm/cytofix kit (BD PharMingen) For surface staining, $5 \times 10^{5}$ cells were washed twice with PBS and stained with peridinin chlorophyll protein-cyanine 7.7conjugated anti-human or mouse CD4 (BD PharMingen) for 30 minutes at $4^{\circ} \mathrm{C}$. To measure the intracellular IL-17 concentrations, the cells were fixed and stained with fluorescein isothiocyanate-conjugated anti-human or antimouse IL-17 monoclonal antibody (BD PharMingen) for 30 minutes at $4^{\circ} \mathrm{C}$. Staining for the isotype controls was performed simultaneously using an isotype control antibody (BD PharMingen). The cells were analyzed on a fluorescence-activated cell sorter (FACS), Calibur (BD). The events were collected and analyzed with FlowJo software (TreeStar, Ashland, OR, USA).

\section{Statistical analysis}

The experimental values are presented as means \pm SD. Statistical significance was determined by analysis of variance (ANOVA) with Bonferroni's post-test correction or Student's $t$-test, using the SPSS program (version 10.0); $P$-values $<0.05$ were considered statistically significant.

\section{Results}

Microarray analysis of IL17A inducible cytokine- and chemokine-related genes

We utilized a microarray to compare the multiple gene expression profiles representative of the FLSs from patients with RA and the IL-17-stimulated FLSs. Table 1 shows the IL-17-inducible cytokine and chemokine genes in the FLSs from patients with RA. Several inflammation-related genes were highly expressed in the IL17-stimulated FLSs from RA patients. Our microarray results indicated that IL-17A induced IL-32 expression (4.3-fold) in FLSs of RA patients (Table 1). Therefore, we examined whether IL-17 and IL-32 have an effect on each other in the inflammatory environment.

\section{IL-17 induced IL-32 expression via NF- $\kappa$ B and PI3kinase in} the FLSs of patients with RA

FLSs obtained from synovial tissue of patients with RA during surgical synovectomy were stimulated by IL-17, and the IL-32 mRNA level was measured. The IL-32 mRNA level was increased in a dose-dependent manner (Figure 1A). The IL-32 mRNA level was decreased by the PI3K inhibitor LY294002 and the NF- $\kappa$ B inhibitor parthenolide, and PI3K and NF- $\kappa \mathrm{B}$ molecules were associated with the IL-17-induced IL-32 production (Figure 1B) [13]. A higher level of IL-32 was expressed by IL-17-stimulated FLSs from patients with RA than from patients with OA. Similarly, NF- $\kappa \mathrm{B}$ and PI3K signal molecules were also more highly expressed in synovium from patients with RA than from patients with OA (Figure 1C).

In the inflammatory condition of RA synovitis, contact between $\mathrm{T}$ cells and FLSs is an important and necessary mechanism [26]. Co-incubation of FLSs from RA patients with $\mathrm{CD}^{+} \mathrm{T}$ cells caused an increase in IL-32 mRNA levels in FLSs (Figure 2A, left panel) and an increase in IL-17 levels in the supernatants of co-cultures (Figure 2A, right panel). When treated with IL-17 blockade antibody

Table 1 Interleukin (IL)-17A-inducible genes as determined by microarray analysis of fibroblast-like synoviocytes (FLSs) from patients with rheumatoid arthritis (RA)

\begin{tabular}{|c|c|c|c|}
\hline Accession numbers & Full name & Symbol & Folds increased \\
\hline NM_000584.2 & interleukin 8 & 118 & 509.5 \\
\hline NM_004591.1 & chemokine (C-C motif) ligand 20 & CCL2O & 382.0 \\
\hline NM_002993.2 & chemokine (C-X-C motif) ligand 6 & CXCL6 & 314.1 \\
\hline NM_000600.1 & interleukin 6 & IL6 & 120.6 \\
\hline NM_001511.1 & chemokine (C-X-C motif) ligand 1 & CXCL1 & 88.6 \\
\hline NM_007115.2 & tumor necrosis factor, alpha-induced protein 6 & TNFAIP6 & 21.1 \\
\hline NM_002089.1 & chemokine (C-X-C motif) ligand 2 & CXCL2 & 20.4 \\
\hline NM_002982.3 & chemokine (C-C motif) ligand 2 & $C C L 2$ & 20.0 \\
\hline NM_005623.2 & chemokine (C-C motif) ligand 8 & CCL8 & 17.5 \\
\hline NM_002421.2 & matrix metallopeptidase 1 & MMP1 & 16.1 \\
\hline NM_001078.2 & vascular cell adhesion molecule 1 & VCAM1 & 15.2 \\
\hline NM_000201.1 & intercellular adhesion molecule 1 & ICAM1 & 9.3 \\
\hline NM_001565.1 & chemokine (C-X-C motif) ligand 10 & CXCL10 & 8.2 \\
\hline NM_001012632.1 & interleukin 32 & $1 / 32$ & 4.3 \\
\hline NM_002006.3 & fibroblast growth factor 2 & FGF2 & 2.7 \\
\hline
\end{tabular}

Bead array gene expression analysis of FLSs and IL-17 activated FLSs from patients with RA (RA FLS) at 12 h. After serum starvation for $24 \mathrm{~h}$, the RA FLSs were stimulated with IL-17A ( $20 \mathrm{ng} / \mathrm{ml})$ or without IL-17A. Total RNA from RA FLSs was used for the Bead array gene expression analysis. The genes differentially expressed between the IL-17 activated RA FLSs and the RA FLSs were identified by ANOVA with a $P$-value $<0.01$. 


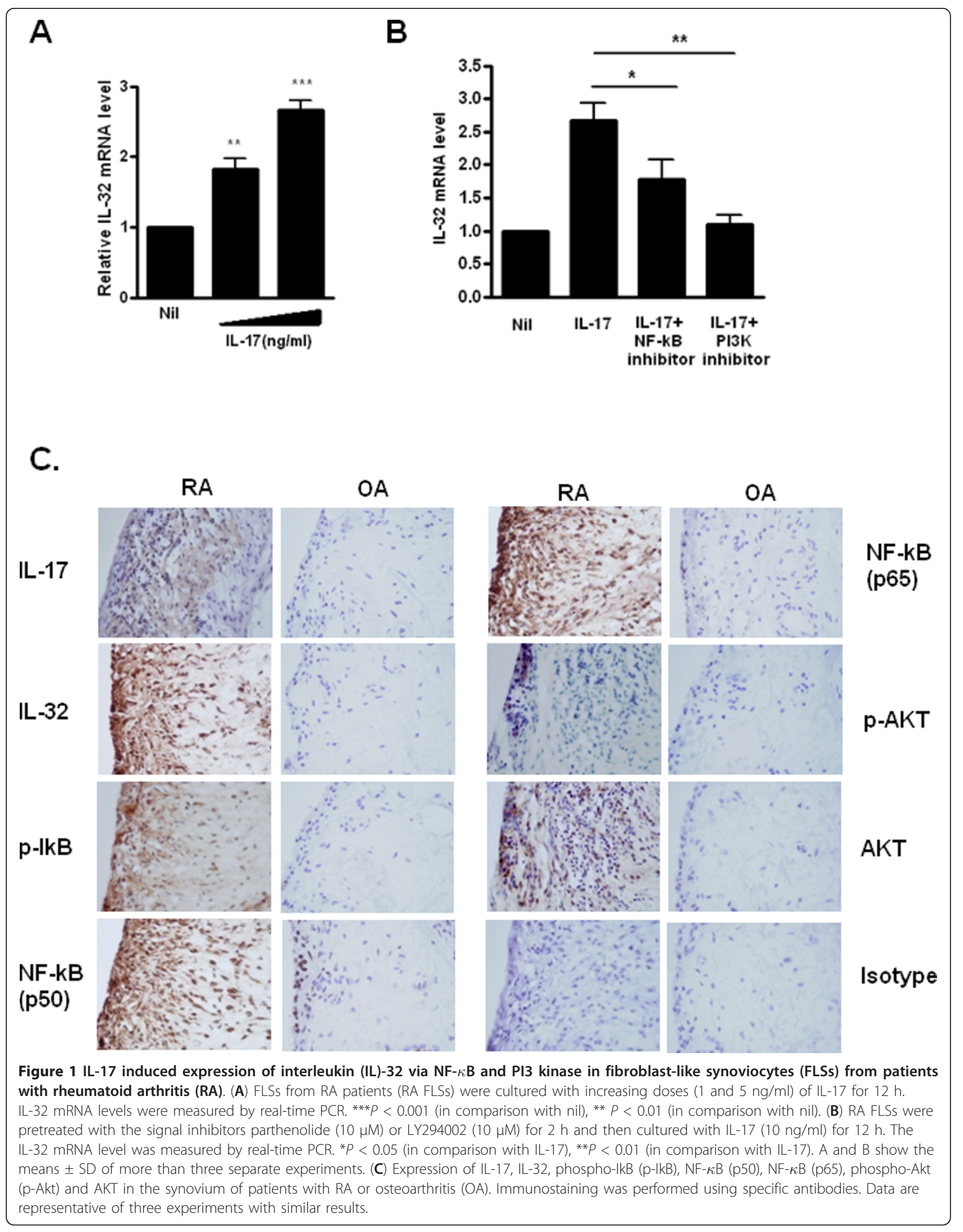


A

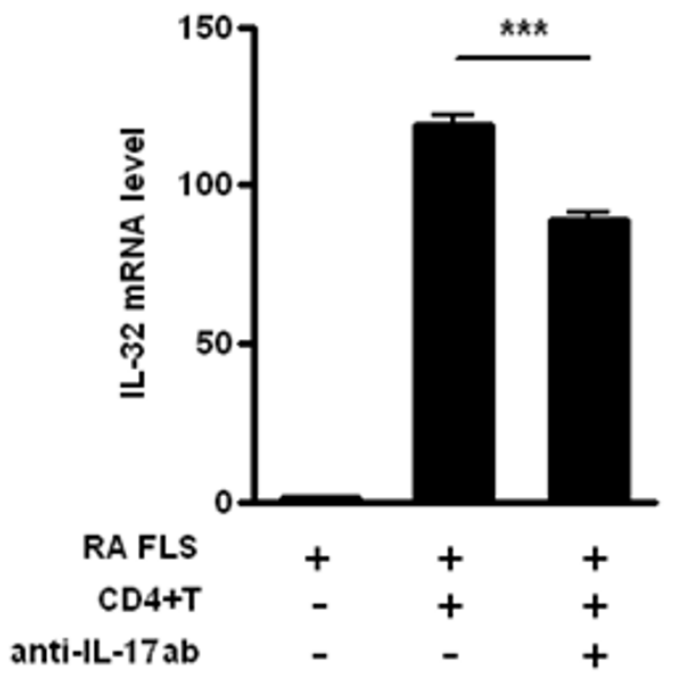

B

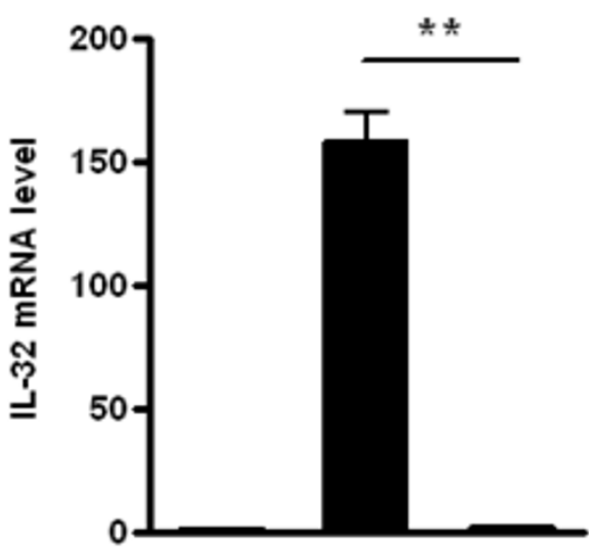

RA FLS

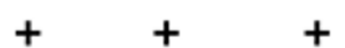

Supernatant of Th17 polarized cell

Supernatant of

Th17 polarized cell

with anti-IL17Ab
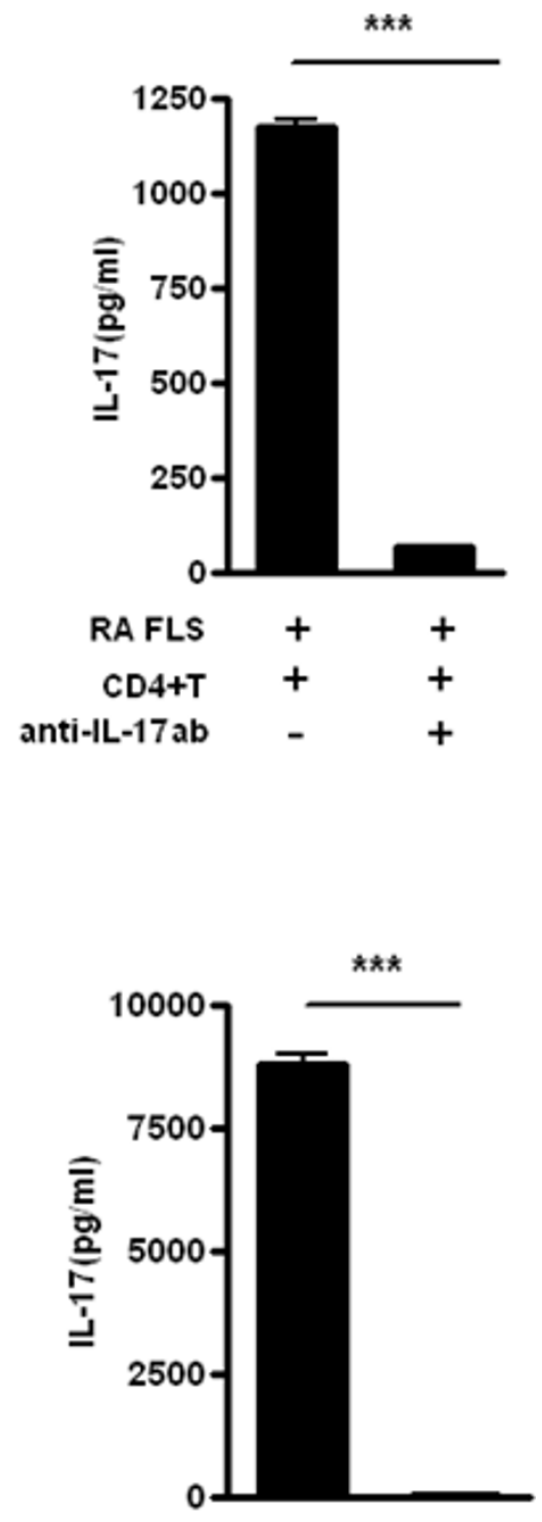

RAFLS $+\quad+$

Supernatant of Th17 polarized cell

Supernatant of

Th17 polarized cell

with anti-IL17Ab

Figure 2 Interleukin (IL)-17/T helper (Th)17-induced IL-32 expression from rheumatoid arthritis (RA) patients. (A) Production of IL-32 by RA fibroblast-like synoviocytes (FLSs) in contact with $\mathrm{CD}^{+} \mathrm{T}$ cells. RA FLSs and $\mathrm{CD} 4^{+} \mathrm{T}$ cells from healthy donors were co-cultured. FLSs $\left(1 \times 10^{5}\right)$ were cultured with CD4 $4^{+} T$ cells $\left(1 \times 10^{6}\right)$ with or without anti-lL-17 blocking antibody $(10 \mu \mathrm{g} / \mathrm{ml})$. IL-17 production was then measured by sandwich ELISA, and the IL-32 mRNA levels of RA FLSs were determined by real-time PCR. ${ }^{* *} P<0.001$ (compared with FLS+CD4 ${ }^{+}$T cells). (B) Induction of RA FLS IL-32 production by the supernatant of Th17-polarized cell cultures. RA FLSs and the culture supernatants of Th17-polarized cells from healthy donors were co-cultured. CD4 ${ }^{+}$T cells were incubated with membrane-bound anti-CD3 antibody $(2 \mu \mathrm{gg} / \mathrm{ml})$, IL-6 (5 ng/ml), IL $1 \beta$ (5 ng/ml), IL-23 (10 $\mathrm{ng} / \mathrm{ml})$, TGF- $\beta(5 \mathrm{ng} / \mathrm{ml})$ with or without an anti-IL-17 blocking antibody incubated for $2 \mathrm{~h}$ before the next incubation) for 3 days to induce Th17. polarization. FLS $\left(1 \times 10^{5}\right)$ were cultured with the culture supernatants of these Th17 polarized cells. IL-17 production was measured by sandwich ELISA and the IL-32 mRNA levels in RA FLSs were determined by real-time PCR. ${ }^{* *} P<0.01$ (compared with FLSs + culture supernatant of Th17 cells), *** $P<0.001$ (compared with FLSs + culture supernatant of Th17 cells). The data are representative of three experiments with similar results. 
under the same conditions, the IL-32 mRNA levels in the FLSs were suppressed (Figure 2A, left panel). The IL-17 levels in the supernatant of co-cultures were also markedly decreased by blocking with anti-IL-17 antibody (Figure 2A, right panel).

To confirm the role of IL-17 of CD4 ${ }^{+} \mathrm{T}$ cells in induction of IL-32 expression in FLSs, the IL-17-rich supernatant from Th17-polarized cells was added to FLSs from RA patients. The IL-32 mRNA level in the FLSs from RA patients was increased in the presence of the Th17-polarized cell supernatant, and blocked with IL-17 blockade antibody (Figure 2B, left panel). Therefore, IL-17 derived from direct contact between FLSs from RA patients and $\mathrm{CD}^{+} \mathrm{T}$ cells (Figure 2A), as well as that secreted by Th17-polarized cells (Figure 2B), can induce IL-32 expression in FLSs from RA patients. In both cases, IL-32 expression was arrested by IL-17 blockade. These results demonstrate that IL-17, an important cytokine in RA, has a direct role in IL-32 expression by the FLSs of RA patients.

\section{IL-32 induced the production of IL-17 in human CD4 ${ }^{+}$ $\mathrm{T}$ cells and differentiation of Th17 cells}

Because IL-32 can induce inflammatory cytokines, we next assessed the IL-17 production by IL-32. To determine whether IL-32 induces IL-17 production, CD4 ${ }^{+} \mathrm{T}$ cells from human PBMCs were cultured with membranebound anti-CD3 antibody to activate TCRs and the expression of IL-17 increased when was stimulated with IL-32 (Figure 3A). CD4 ${ }^{+} \mathrm{T}$ cells from healthy donors were differentiated using anti-CD3, anti-CD28, anti-IL-4, and anti-IFN- $\gamma$, antibodies, and IL- $1 \beta$ and IL-6. FACS analysis showed an increase in IL-17-expressing cells after IL-32 stimulation (Figure 3B). In addition, IL-17 mRNA levels in human $\mathrm{CD}^{+} \mathrm{T}$ cells were increased by IL-32 stimulation, and expression of ROR $\gamma \mathrm{t}$, a transcription factor for Th17 differentiation, was also increased by IL-32 stimulation (Figure 3C). Th17 cells might secrete increased IL-17 in the presence of IL-32. Indeed, recombinant human IL-32 promoted IL-17 production (Figure 3D). These results suggest that IL-32 production is affected by IL-17 stimulation, and that IL-32 plays a role in both Th17 cell differentiation and IL-17 secretion.

\section{IL-32 induced IL-17 production in autoimmune arthritis mouse models}

IL-17 and IL-32 interact in human FLSs and T cells from patients with RA. To confirm this interaction in autoimmune arthritis mouse models, splenic $\mathrm{CD}^{+} \mathrm{T}$ cells of type-II collagen-induced mice (CIA) were cultured with anti-CD3 or anti-CD3 with IL-32 $\alpha$. Increased IL-17 secretion was observed with IL-32 stimulation in this animal model (Figure 4A). In addition, we examined whether IL-32-treated Th17 polarized cells secreted more IL-17, in a manner similar to that observed in the human RA condition. $\mathrm{CD}^{+} \mathrm{T}$ cells and irradiated CD4- $\mathrm{T}$ cells (as antigen presenting cells) from CIA mice at 5 weeks after immunization were co-cultured with Th17 polarizing condition with/without CII/IL-32. A second challenge with antigen increased $\mathrm{CD} 4{ }^{+} \mathrm{IL}_{-1} 7^{+}$cells (Figure 4B). Moreover, IL-32 induced the secretion of IL-17 (Figure 4C) and increased the expression of IL-17 mRNA (Figure 4D). These results show that a second collagen challenge induced IL-17 secretion, and that IL-32 also promoted IL-17 mRNA expression and cytokine secretion.

Next, we investigated whether IL-17 and IL-32 have a role in bony erosion in RA mouse models. To correlate the location of cytokine expression and osteoclastogenesis, the synovium of both CIA and IL-1Ra knockout (KO) mice were analyzed for IL-17, IL-32 and TRAP expression, and stained with H\&E (Figure 4E). Damaged bone areas demonstrated TRAP-positive cells. IL-32 and IL-17 were co-localized near the TRAP-positive areas between the cartilage and tarsal bone of both mouse models (Figure 4E). Thus, in two RA animal models, IL-32 induced IL-17 production, and IL-32 and IL-17 were expressed in areas of osteoclast differentiation.

\section{IL-17 and IL-32 synergistically induced osteoclastogenesis}

Osteoclast precursors were cultured in the presence of IL-17 and/or IL-32 with M-CSF, a cytokine involved in the survival and proliferation of osteoclasts. After 15 days culture, IL-17 or IL-32 induced the formation of multinucleated TRAP positive cells as shown in Figure 5A. The numbers of IL-17 or IL-32-stimulated TRAP-positive MNCs were increased compared to those observed with M-CSF alone. Although RANKL is well known as a crucial factor for osteoclast differentiation, these results revealed that IL-32 and IL-17 are involved in RANKLindependent mechanisms of osteoclastogenesis. In addition, the simultaneous stimulation by IL-17 and IL-32 induced an increased number of multinucleated TRAPpositive cells (Figure 5B). These findings imply that IL-17 and IL-32 synergistically induced osteoclastogenesis from osteoclast precursor cells in the absence of a RANKLRANK interaction. Osteoclast markers, such as calcitonin receptor, cathepsin K, TRAP and MMP9 mRNAs, were increased by IL-17 or IL-32 as much as that observed with RANKL stimulation (positive control). IL-17 and IL32 had a synergistic effect on induction of osteoclast markers at the mRNA level in a RANKL-independent manner (Figure 5C). In conditions that included IL-32 and IL-17 without RANKL, resorption pit formation was not observed. When treated with RANKL, IL-32 and IL-17 synergistically accelerated the osteoclast resorption activity compared with IL-32 or IL-17 alone (Figure 5D). These results indicate that IL-32 and IL-17 could synergistically induce osteoclastogenesis independent of 


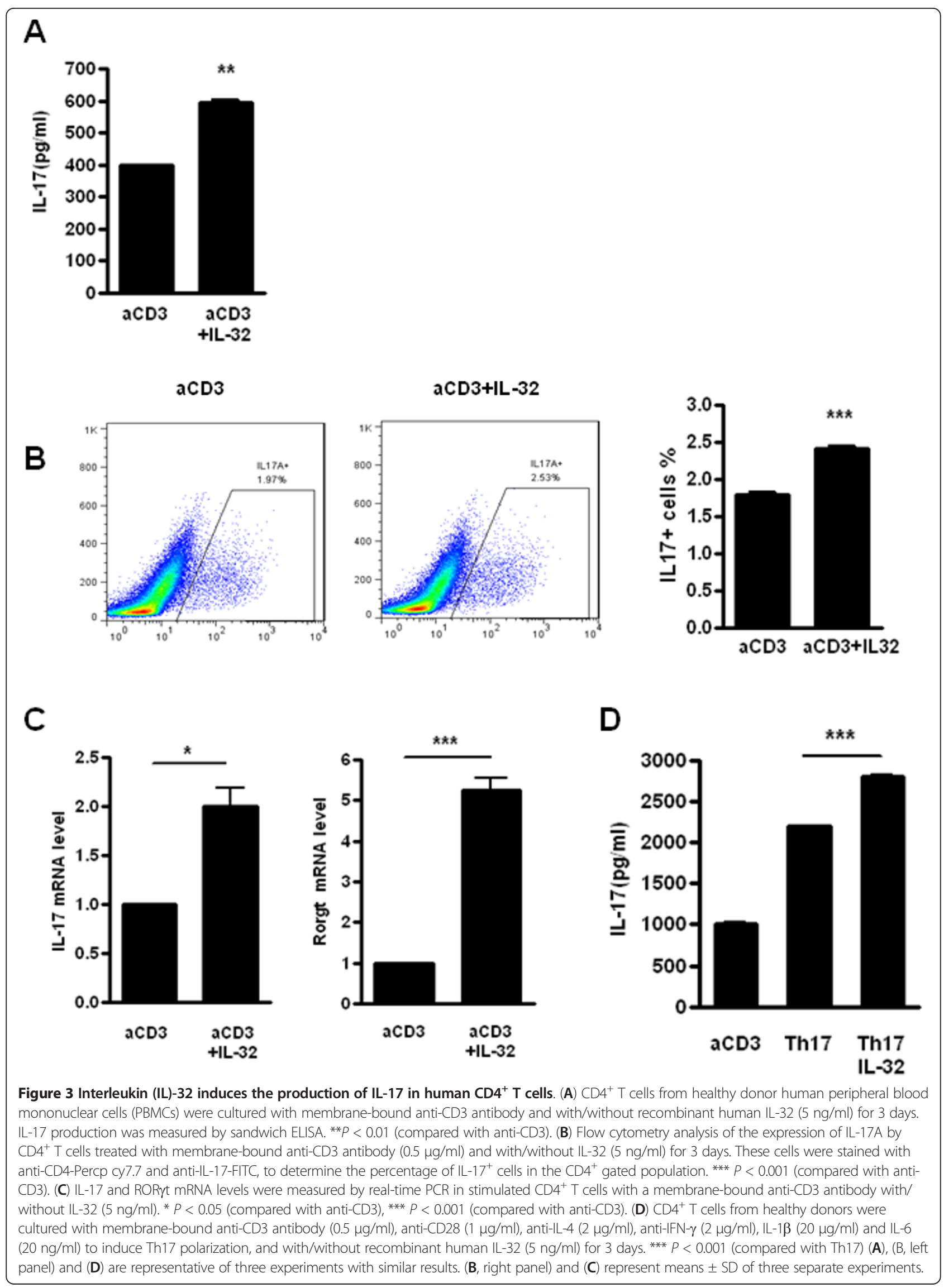




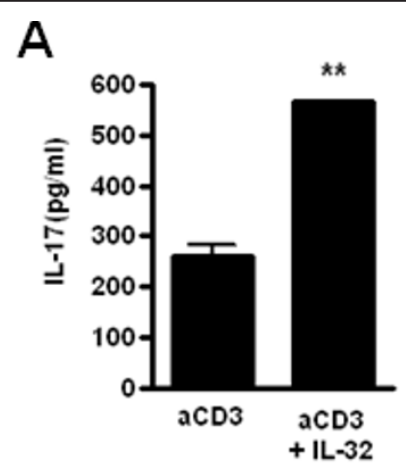

B

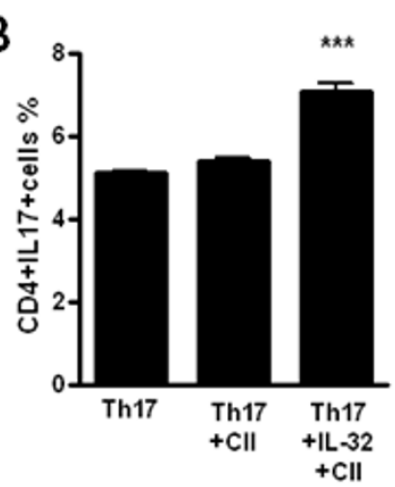

C

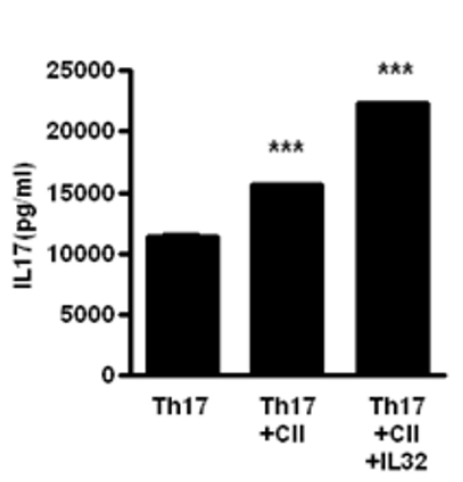

D

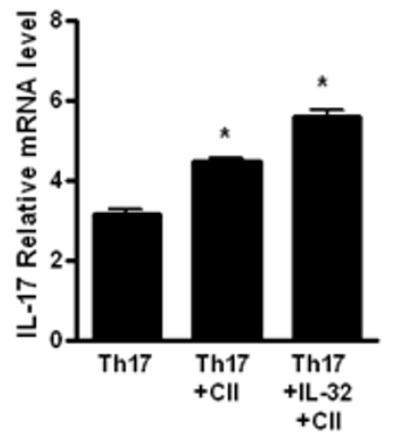

E

$$
\text { IL }-32
$$

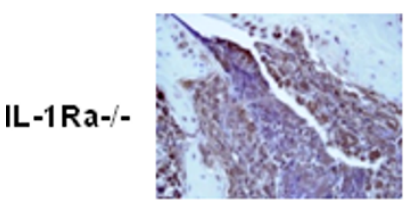

CIA
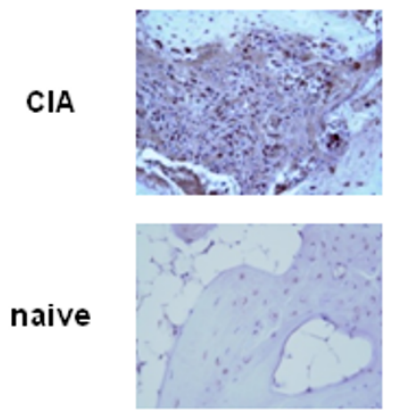

$x 400$
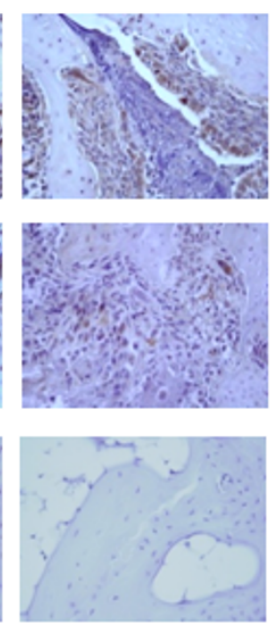

$x 400$
Iso
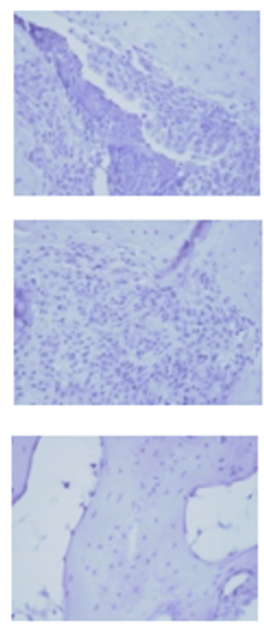

$x 400$
TRAP
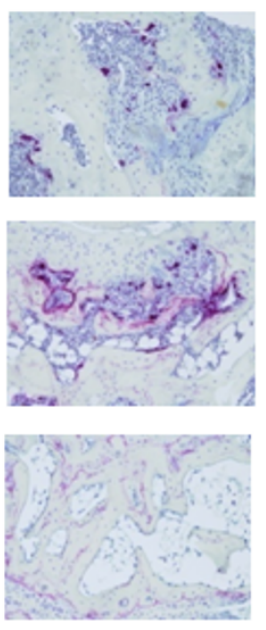

$\times 200$
H\&E
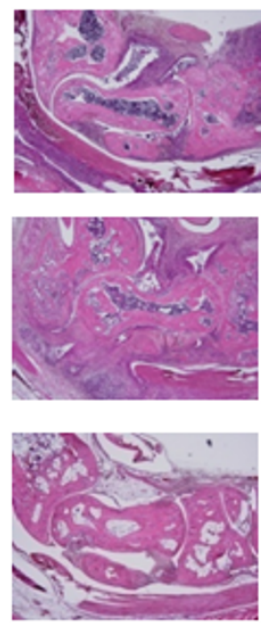

$x 40$

Figure 4 Interleukin (IL)-32 induces IL-17 production in an autoimmune arthritis mouse model. (A) CD4 ${ }^{+} \mathrm{T}$ cells were isolated from the spleens of collagen-induced arthritis (CIA) mice, an autoimmune arthritis model. The cells were cultured with membrane-bound anti-CD3 (0.5 $\mu \mathrm{g} / \mathrm{ml})$ and with/without IL-32 $(5 \mathrm{ng} / \mathrm{ml})$ for 3 days. IL-17 production was measured by sandwich ELISA. ** $P<0.01$ (compared with anti-CD3). (B-D) Expression of IL-17A by CD4 ${ }^{+}$T cells cultured under Th17-polarizing conditions for 3 days: anti-CD3 $(0.5 \mu \mathrm{g} / \mathrm{ml})$, anti-CD28 (1 $\left.\mu \mathrm{g} / \mathrm{ml}\right)$, antiIFN-gamma $(2 \mu \mathrm{g} / \mathrm{ml})$, anti-lL-4 $(2 \mu \mathrm{g} / \mathrm{ml})$, anti-lL-2 $(2 \mu \mathrm{g} / \mathrm{ml})$, IL-6 $(20 \mathrm{ng} / \mathrm{ml})$, TGF-beta1 $(2 \mathrm{ng} / \mathrm{ml})$ with/without IL-32 $(5 \mathrm{ng} / \mathrm{ml})$ and/or type II collagen with irradiated antigen-presenting cells, and then stimulated for $4 \mathrm{~h}$ with PMA and ionomycin, followed by intracellular cytokine staining. The percentage of $1 \mathrm{~L}-17^{+}$cells in the $\mathrm{CD}^{+}$gated population (B), *** $P<0.001$ (compared with Th17) and cytokine level (C) were measured by sandwich ELISA, *** $P<0.001$ (compared with Th17) and mRNA levels (D) were measured by real-time PCR, * $P<0.05$ (compared with Th17). B-D are representative of three experiment with similar results. (E) Expressions of IL-17, IL-32 and tartrate-resistant acid phosphatase (TRAP) in the synovium of the CIA and IL-1Ra-knock-out (KO) mice, two autoimmune arthritis mouse models. The results shown are representative of five experiments with similar results. 


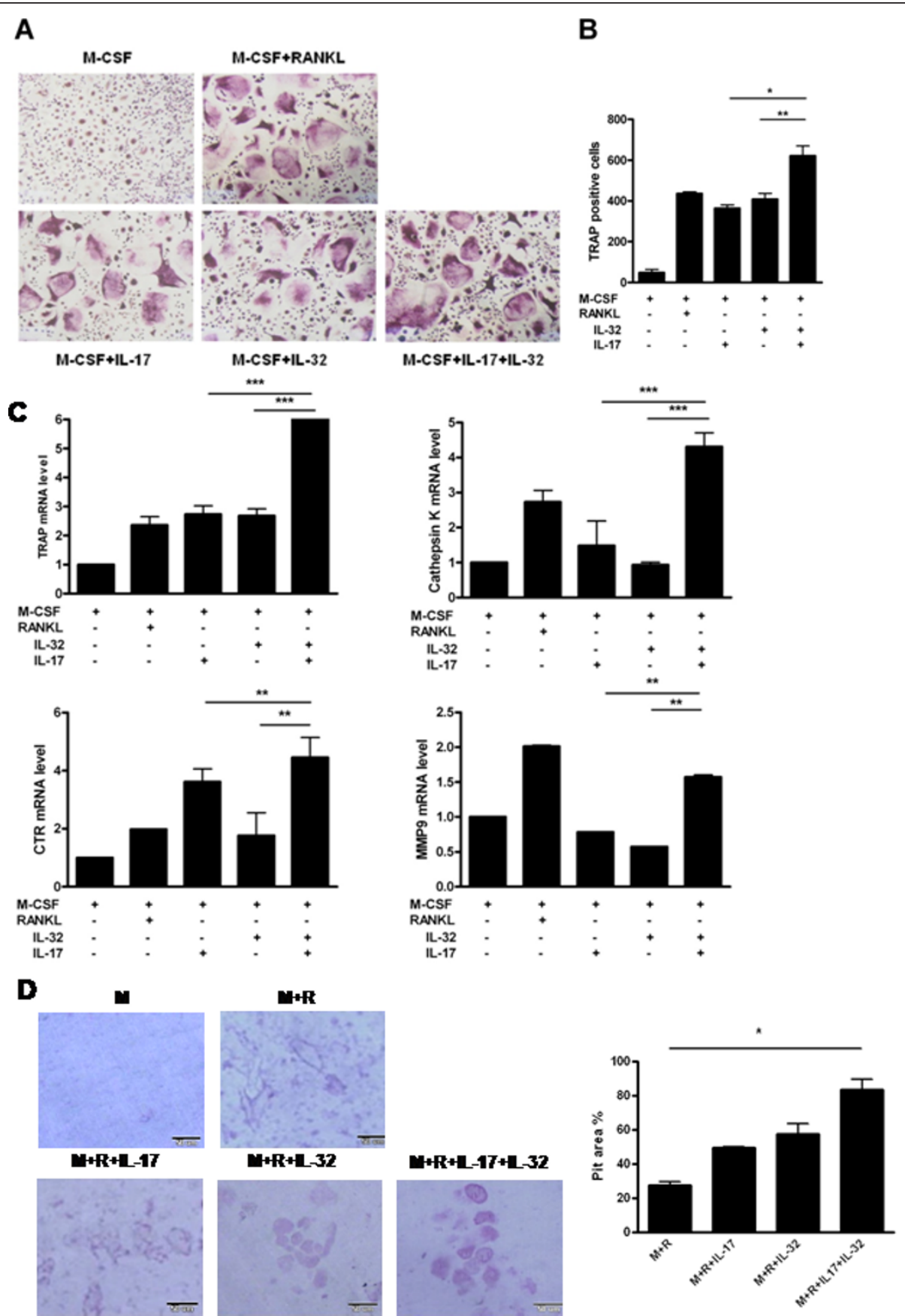

Figure 5 Interleukin (IL)-17 and IL-32 synergistically induce osteoclastogenesis. (A) Tartrate-resistant acid phosphatase (TRAP) staining for identification of osteoclasts. Osteoclast precursors were cultured in the presence of IL-17 (0.1 ng/ml) or/and IL-32 $(5 \mathrm{ng} / \mathrm{ml})$ with macrophage colony-stimulating factor (M-CSF; $25 \mathrm{ng} / \mathrm{ml}$ ). The receptor activator of the nuclear factor kappa-B ligand (RANKL; $30 \mathrm{ng} / \mathrm{ml}$ )-treated group was the positive control. The medium and stimulus were changed every 3 days. After 15 days, the cells were stained for TRAP activity. (B) Numbers of multinucleated TRAP-positive cells per well. TRAP-positive cells containing two or more nuclei were scored as osteoclasts. TRAP-positive cells were counted three times by blind scoring. ${ }^{*} P<0.05$ (compared with M-CSF+IL-32+IL-17), ${ }^{* *} P<0.01$ (compared with M-CSF+IL-32+IL-17). (C) The mRNAs of TRAP, Cathepsin K, calcitonin receptor (CTR) and matrix metallopeptidase 9 (MMP9), as osteoclast markers were quantified by real-time PCR. ${ }^{*} P<0.05$ (compared with M-CSF+IL-32+IL-17), ${ }^{*} P<0.01$ (compared with M-CSF+IL-32)+IL-17), ***P< 0.001 (compared with MCSF+IL-32+IL-17). (D) Formation (left) and percent area (right) of resorption pits by osteoclasts on dentine discs. Cell culture was performed as described with dentine discs in 96-well plates. The osteoclast precursors were cultured in the presence of $\mathrm{LL}-17(1 \mathrm{ng} / \mathrm{ml}) \mathrm{or} \mathrm{IL}-32(5 \mathrm{ng} / \mathrm{ml})$ with M-CSF $(25 \mathrm{ng} / \mathrm{ml})$ and RANKL $(30 \mathrm{ng} / \mathrm{ml})$. At day 21, the cells were removed from dentine. Resorption area was evaluated by light microscopy and measured using the image analysis software. (A), (B) and (D) are representative of two or three experiments with similar results. (C) Means \pm SD of more than three separate experiments. 
RANKL, and could synergistically induce resorption by osteoclasts in a RANKL-dependent manner.

\section{Discussion}

IL-32 is a novel cytokine that has been reported to be an important player in the innate and adaptive immune response. Innate immune stimulation by the ligands of toll-like receptors (TLRs) can induce the expression of IL-32 in FLSs [27]. Recently, the expression of IL-32 was demonstrated in Mycobacterium tuberculosis infections, inflammatory bowel disease and influenza A virus infection, in addition to that in autoimmune disease. However, more research is required to explain the function of IL-32 in inflammatory disorders.

The expression and regulation of cytokines involved in RA pathogenesis have been studied extensively over the past two decades, which is possibly due to the relative ease of analyzing the synovium of RA patients. These investigations have broadened and deepened our understanding of the roles of several cytokines in RA pathogenesis. Furthermore, some studies, most notably those focusing on TNF $\alpha$, IL-1, and IL-6, have shown promising ways to block the cytokines involved in RA pathogenesis. It is important to characterize the individual cytokines in relation to the pathogenesis of RA and understanding the interaction and cross-talk between these cytokines is critical. Cytokines can amplify other inflammatory cytokines and then compensate for this by increasing the production of pro-inflammatory cytokines. For instance, it has been shown that IL-32 is a potent inducer of TNF- $\alpha$ [28]. In addition, TNF- $\alpha$ is a potent inducer of endogenous IL-32 expression, and IL-32 itself prolongs TNF- $\alpha$ production, thus inducing an important autoinflammatory loop [20]. IL-17 is critical for RA pathogenesis and can induce increased expression of TNF $\alpha$ [25]. To-date, a direct interaction between IL-17 and IL-32 has not been reported, although one study implied their interaction by determining that these cytokines have common signal intermediates, p300 and DAPK-1 [22]. Another study demonstrated that IL-32 $\gamma$-stimulated DCs could induce a Th17 response when co-cultured with $\mathrm{CD} 4^{+} \mathrm{T}$ cells [29], implying that IL-32 affects IL-17 production independent of TNFa.

In this study, we examined the synergistic expressions of IL-17 and IL-32 and their possible interactions. Using microarray analysis, we showed that IL-17 induced gene expression in the FLSs of patients with RA (Table 1), and that IL-32 mRNA levels increased in a dose-dependent manner (Figure 1A). Because IL-32 $\alpha$ mRNA expression depends on the PI3-kinase/NF- $\kappa$ B systems in human colonic subepithelial myofibroblasts [30], we investigated whether IL-17 is also dependent on the NF- $\kappa \mathrm{B} / \mathrm{PI} 3$-kinase pathways in FLSs. of patients with RA. It was confirmed that this induction occurred through the NF- $\kappa \mathrm{B}$ and PI3kinase pathways by using specific inhibitors to block these pathways (Figure 1B). IL-32 induction by TNF- $\alpha$, is mediated via the Syk/Protein Kinase $\mathrm{C} \delta / \mathrm{JNK}$ signaling pathways [31]. The transcription and secretion of proinflammatory and anti-inflammatory cytokines have the complex signal pathways inside cells.

IL-32 expression in the synovium from patients with RA than in those with OA was increased (Figure 1C). The expression of IL-32 in synovium of patients with RA was also co-localized with IL-17, NF- $\kappa \mathrm{B}$, and PI3K. This result suggests that FLSs from patients with RA could interact with T cells, especially IL-17-producing Th17 cells, and direct contact could be important for this interaction.

We observed that co-culture of FLSs with $\mathrm{CD} 4^{+} \mathrm{T}$ cells resulted in production of IL-17, which caused IL32 expression in FLSs. This IL-32 induction was partially inhibited by blocking with anti-IL-17 antibodies (Figure 2A). Coculture of FLSs with the IL-17-rich supernatant from Th17-polarized cells resulted in a markedly increased IL-32 production by FLSs from RA patients. To determine whether IL-17 had a direct effect, we used the supernatant of the IL-17 blocked Th17 polarized cells. These results suggested that IL-17 is directly related to IL-32 production with the interaction of $\mathrm{CD}^{+}{ }^{+} \mathrm{T}$ cells and FLSs. Since IL-17 was generally not produced by FLSs, IL-17 secreted from CD $4^{+} \mathrm{T}$ cells might induce IL-32 expression in FLSs. IL-17-rich supernatant induced more IL-32 mRNA expression. Although these inductions were arrested by anti-IL-17 antibody, IL-17-rich supernatant with anti-IL-17 antibody was more reduced in IL-32 mRNA expression than $\mathrm{CD} 4^{+} \mathrm{T}$ cell with anti-IL-17 antibody. The reason could be due to the production of other factors than IL17 by cell-cell contact. The identification of these factors would be of interest for future study. It has been reported that TNF $\alpha$ exhibits potent induction of IL-32 secretion in FLSs of patients with RA. One study showed that IL-17 is related to IL-32 expression in FLSs of RA patients [19]. In contrast to our results, this study reported that both IL-17A and IL-17F induced IL-32 to only a small extent. In addition, IL-17 attenuated the IL-32 expression induced by TNF $\alpha$. IL-17, an important proinflammatory cytokine, is unlikely to have a paradoxically negative feedback in the autoinflammatory loop between IL-32 and TNF $\alpha$. Therefore, we suggest that our results, showing that IL-17 amplified IL-32 expression in FLSs of patients with RA, are logically acceptable.

Human IL-32 recombinant protein has been utilized in mice $[18,29]$, and so we determined whether IL-32 could induce IL-17 production in an autoimmune arthritis mouse model. Using ELISA and FACS analysis, we showed that IL-32 induced high IL-17 expression in splenic $\mathrm{CD}_{4}^{+} \mathrm{T}$ cells of CIA mice. IL-32 induced the 
differentiation of $\mathrm{CD}^{+}{ }^{+} \mathrm{T}$ cells of RA model mice to Th17 cells and promoted IL-17 production. In addition, immunohistochemistry staining indicated that IL-17, IL32 and TRAP were co-localized in the joints of CIA and IL-1R antagonist-deficient mice. These results suggest that an interaction between IL-32 and IL-17 exists in both autoimmune arthritis diseases and animal models, contributing to accelerated inflammation and bone destruction.

Next, we revealed that IL-17 and IL-32 have synergistic roles in osteoclastogenesis. Osteoclasts are multinucleated cells that are responsible for bone resorption and are derived from hematopoietic precursor cells that circulate in the blood. It is currently thought that two critical factors (M-CSF and RANKL) supplied by osteoblasts are essential for the differentiation and maturation of osteoclast precursors. Moreover, some researchers have reported that a direct interaction between osteoclast progenitors and osteoblasts is required for IL-17-induced osteoclastogenesis [5]. IL-17 dose-dependently induced the expression of osteoclast differentiation factor (ODF) mRNA in osteoblasts. IL-32 also promotes osteoclast differentiation and the expression of several specific markers of osteoclasts such as NFATc1, OSCAR and cathepsin K [23]. To determine whether IL-17 and IL-32 have a synergistic effect in osteoclastogenesis, we cultured osteoclast precursors with IL-17 and/or IL-32. IL-17 and IL-32 accelerated osteoclastogenesis compared with IL-17, IL-32 or RANKL stimulation alone (Figure 5A, B). We also examined the effect of IL-17 and IL-32 on osteoclast differentiation in the presence of anti-RANKL or anti-OPG antibodies, and observed that osteoclastogenesis occurred in a manner that was independent of RANKL or OPG (data not shown). In addition, we investigated whether IL17- and IL-32-stimulated osteoclastogenesis was affected by TNF $\alpha$. Although we detected TNF $\alpha$ in the supernatant of IL-17- and IL-32-stimulated differentiated osteoclasts, blocking with anti-TNF $\alpha$ showed no effect (data not shown). Therefore, osteoclastogenesis associated with IL-17 and IL-32 was not dependent on RANKL or TNF $\alpha$.

To determine the resorption activity of osteoclasts induced by IL-32 and Il-17, we performed a resorption pit assay using dentine slices. When IL-32 and IL-17 were assayed in the absence of RANKL, no resorption pit formation was observed. Moreover, we confirmed that RANKL is essential for osteoclast resorption activity. When treated with RANKL, IL-32 and IL-17 synergistically accelerated osteoclast resorption activity compared with IL-32 or IL-17 alone. We deduced that IL-32 and IL-17 could synergistically induce osteoclastogenesis independent of RANKL, and were synergistically involved in the RANKL-dependent resorption function of osteoclasts.

FLSs and $\mathrm{CD}^{+} \mathrm{T}$ cells are found in close proximity in synovial joints. We suggested that IL-17 and IL-32 could stimulate the reciprocal production of each other, and amplify inflammatory reactions. In this model, the two cytokines synergistically stimulate osteoclastogenesis independently of RANKL, and might increasingly induce bony erosion and osteopenia together with RANKL, thus participating in the inflammation associated with RA. Therefore, interruption of IL-17 and IL-32 might be a therapeutic target for treatment of inflammatory arthritis.

\section{Conclusions}

This report is the first to show that IL-17 induces IL-32 cytokine expression through the NF- $\kappa \mathrm{B}$ and PI3-kinase signal pathways in FLSs of patients with RA. IL-17 is produced by Th17 cells that differentiate from $\mathrm{CD}^{+} \mathrm{T}$ cells in patients with RA. In the joint environment of inflammation, $\mathrm{CD}^{+} \mathrm{T}$ cells and FLSs interact with each other by direct contact and cytokine secretion, and this interaction amplifies the expression of IL-17 and IL-32. IL-32 induced high levels of IL-17 expression in the splenic $\mathrm{CD}^{+}{ }^{+} \mathrm{T}$ cells of CIA mice. Co-localization of IL-32, IL-17 and TRAP suggested the possibility of their functional interaction in autoimmune arthritis models. Both IL-17 and IL-32 induce the gene markers CTR, capthepsin K, TRAP and MMP9, which are all related with osteoclastogenesis. IL-17 and IL-32 have a synergistic effect on the expression of these genes in CD $4^{+} \mathrm{T}$ cells and FLSs. IL-17 and IL-32 have a reciprocal influence on each other's production, and enhance osteoclastogenesis in the synovium of patients with RA.

\section{Abbreviations}

ANOVA: analysis of variance; CIA: collagen-induced arthritis; CII: type II collagen; Cp: crossing point; DC: dendritic cell; FBS: fetal bovine serum; ELISA: enzyme-linked immunosorbent assay; FACS: fluorescence-activated cell sorter; FLS: fibroblast-like synoviocyte; H\&E: hematoxylin and eosin; IFN: interferon; IL: interleukin; M-CSF: macrophage colony-stimulating factor; MMP: matrix metalloproteinase; MNC: multinucleated cell; NF-kB: nuclear factor-KB; NK: natural killer; OA: osteoarthritis; PI3K: phosphatidylinositol (PI)-3 kinase; PBMC: peripheral blood mononuclear cell; PCR: polymerase chain reaction; RA: rheumatoid arthritis; RANKL: receptor activator of nuclear factor kappa-B ligand; RBC: red blood cell; SDF: stromal cell-derived factor; TCR: T cell receptor; Th: T helper; TLR: toll-like receptor; TNF: tumor necrosis factor; TRAP, tartrate-resistant acid phosphatase.

\section{Acknowledgements}

This work was supported by the Basic Science Research Program through the National Research Foundation of Korea (NRF), funded by the Ministry of Education, Science and Technology (grant number 2009-0081791) and by a grant of the Korea Health Technology R\&D Project, Ministry for Health, Welfare \& Family Affairs, Republic of Korea (grant number A092258).

\section{Author details}

'The Rheumatism Research Center, Catholic Research Institute of Medical Science, The Catholic University of Korea, 505 Banpo-dong, Seocho-gu, Seoul 137-701, South Korea. ${ }^{2}$ Department of Internal Medicine Inje University Ilsan Paik Hospital, Juhwa-ro 170, Ilsanseo-gu, Goyang-si, Gyeonggi-do 411-706, South Korea. ${ }^{3}$ Conversant Research Consortium in Immunologic disease, Seoul St. Mary's Hospital, College of Medicine, The Catholic University of Korea, 505 Banpo-dong, Seocho-gu, Seoul 137-701, South Korea. 


\section{Authors' contributions}

YMM, BYY and MLC contributed to the conception and design, acquisition of data, analysis and interpretation of data, drafting of the article and final approval of the submitted manuscript. OHJ, KWK, KSP and SHP contributed to immunohistochemistry and TRAP staining. YJW, SYL, YMH, JSL and HYK helped with the PCR, FACS and ELISA. YMH contributed cell culture. All authors approved the final manuscript.

\section{Competing interests}

The authors declare that they have no competing interests in relation to this manuscript.

Received: 20 June 2012 Revised: 30 July 2012

Accepted: 25 October 2012 Published: 13 November 2012

\section{References}

1. Bucala R, Ritchlin C, Winchester R, Cerami A: Constitutive production of inflammatory and mitogenic cytokines by rheumatoid synovial fibroblasts. J Exp Med 1991, 173:569-574.

2. Van Boxel JA, Paget SA: Predominantly T-cell infiltrate in rheumatoid synovial membranes. N Engl J Med 1975, 293:517-520.

3. Yamamura Y, Gupta R, Morita Y, He X, Pai R, Endres J, Freiberg A, Chung K, Fox DA: Effector function of resting T cells: activation of synovial fibroblasts. J Immunol 2001, 166:2270-2275.

4. Langrish CL, Chen Y, Blumenschein WM, Mattson J, Basham B, Sedgwick JD, McClanahan T, Kastelein RA, Cua DJ: IL-23 drives a pathogenic T cell population that induces autoimmune inflammation. J Exp Med 2005, 201:233-240.

5. Kotake S, Udagawa N, Takahashi N, Matsuzaki K, Itoh K, Ishiyama S, Saito S, Inoue K, Kamatani N, Gillespie MT, Martin TJ, Suda T: IL-17 in synovial fluids from patients with rheumatoid arthritis is a potent stimulator of osteoclastogenesis. J Clin Invest 1999, 103:1345-1352.

6. Lubberts $E$, van den Bersselaar L, Oppers-Walgreen B, Schwarzenberger $P$, Coenen-de Roo CJ, Kolls JK, Joosten LA, van den Berg WB: IL-17 promotes bone erosion in murine collagen-induced arthritis through loss of the receptor activator of NF-kappa B ligand/osteoprotegerin balance. J Immunol 2003, 170:2655-2662.

7. Kirkham BW, Lassere MN, Edmonds JP, Juhasz KM, Bird PA, Lee CS, Shnier R, Portek IJ: Synovial membrane cytokine expression is predictive of joint damage progression in rheumatoid arthritis: a two-year prospective study (the DAMAGE study cohort). Arthritis Rheum 2006, 54:1122-1131.

8. Hwang SY, Kim JY, Kim KW, Park MK, Moon Y, Kim WU, Kim HY: IL-17 induces production of IL- 6 and IL-8 in rheumatoid arthritis synovial fibroblasts via NF-kappaB- and PI3-kinase/Akt-dependent pathways. Arthritis Res Ther 2004, 6:R120-128.

9. Cho ML, Jung YO, Kim KW, Park MK, Oh HJ, Ju JH, Cho YG, Min JK, Kim Sl, Park SH, Kim HY: IL-17 induces the production of IL-16 in rheumatoid arthritis. Exp Mol Med 2008, 40:237-245.

10. Kim HR, Cho ML, Kim KW, Juhn JY, Hwang SY, Yoon CH, Park SH, Lee SH, Kim HY: Up-regulation of IL-23p19 expression in rheumatoid arthritis synovial fibroblasts by IL-17 through PI3-kinase-, NF-kappaB- and p38 MAPK-dependent signalling pathways. Rheumatology (Oxford) 2007, 46:57-64.

11. Agarwal $S$, Misra R, Aggarwal A: Interleukin 17 levels are increased in juvenile idiopathic arthritis synovial fluid and induce synovial fibroblasts to produce proinflammatory cytokines and matrix metalloproteinases. J Rheumatol 2008, 35:515-519.

12. Dahl CA, Schall RP, He HL, Cairns JS: Identification of a novel gene expressed in activated natural killer cells and T cells. J Immunol 1992, 148:597-603.

13. Heinhuis B, Koenders MI, van de Loo FA, Netea MG, van den Berg WB, Joosten LA: Inflammation-dependent secretion and splicing of IL-32 \{gamma\} in rheumatoid arthritis. Proc Natl Acad Sci USA 2011, 108:4962-4967.

14. Shioya M, Nishida A, Yagi Y, Ogawa A, Tsujikawa T, Kim-Mitsuyama S, Takayanagi A, Shimizu N, Fujiyama Y, Andoh A: Epithelial overexpression of interleukin-32alpha in inflammatory bowel disease. Clin Exp Immunol 2007, 149:480-486.

15. Cagnard N, Letourneur F, Essabbani A, Devauchelle V, Mistou S, Rapinat A, Decraene C, Fournier C, Chiocchia G: Interleukin-32, CCL2, PF4F1 and GFD10 are the only cytokine/chemokine genes differentially expressed by in vitro cultured rheumatoid and osteoarthritis fibroblast-like synoviocytes. Eur Cytokine Netw 2005, 16:289-292.

16. Netea MG, Azam T, Ferwerda G, Girardin SE, Walsh M, Park JS, Abraham E, Kim JM, Yoon DY, Dinarello CA, Kim SH: IL-32 synergizes with nucleotide oligomerization domain (NOD) 1 and NOD2 ligands for IL-1 beta and IL-6 production through a caspase 1-dependent mechanism. Proc Natl Acad Sci USA 2005, 102:16309-16314.

17. Dinarello CA, Kim SH: IL-32, a novel cytokine with a possible role in disease. Ann Rheum Dis 2006, 65(Suppl 3):iii61-64.

18. Joosten LA, Netea MG, Kim SH, Yoon DY, Oppers-Walgreen B, Radstake TR, Barrera P, van de Loo FA, Dinarello CA, van den Berg WB: IL-32, a proinflammatory cytokine in rheumatoid arthritis. Proc Natl Acad Sci USA 2006, 103:3298-3303.

19. Zrioual $S$, Ecochard R, Tournadre A, Lenief $V$, Cazalis MA, Miossec $P$ : Genome-wide comparison between IL-17A- and IL-17F-induced effects in human rheumatoid arthritis synoviocytes. J Immunol 2009, 182:3112-3120.

20. Heinhuis B, Koenders MI, van Riel PL, van de Loo FA, Dinarello CA, Netea MG, van den Berg WB, Joosten LA: Tumour necrosis factor alphadriven IL-32 expression in rheumatoid arthritis synovial tissue amplifies an inflammatory cascade. Ann Rheum Dis 2011, 70:660-667.

21. Jung MY, Son MH, Kim SH, Cho D, Kim TS: IL-32gamma induces the maturation of dendritic cells with Th1- and Th17-polarizing ability through enhanced IL-12 and IL-6 production. J Immunol 2011, 186:6848-6859.

22. Turner-Brannen E, Choi KY, Arsenault R, El-Gabalawy H, Napper S, Mookherjee N: Inflammatory cytokines IL-32 and IL-17 have common signaling intermediates despite differential dependence on TNF-receptor 1. J Immunol 2011, 186:7127-7135.

23. Mabilleau G, Sabokbar A: Interleukin-32 promotes osteoclast differentiation but not osteoclast activation. PLoS One 2009, 4:e4173.

24. Kim YG, Lee CK, Oh JS, Kim SH, Kim KA, Yoo B: Effect of interleukin32 gamma on differentiation of osteoclasts from CD14+ monocytes. Arthritis Rheum 2010, 62:515-523.

25. Astry B, Harberts E, Moudgil KD: A cytokine-centric view of the pathogenesis and treatment of autoimmune arthritis. J Interferon Cytokine Res 2011, 31:927-940.

26. McInnes IB, Leung BP, Liew FY: Cell-cell interactions in synovitis. Interactions between T lymphocytes and synovial cells. Arthritis Res 2000, 2:374-378.

27. Alsaleh G, Sparsa L, Chatelus E, Ehlinger M, Gottenberg JE, Wachsmann D, Sibilia J: Innate immunity triggers IL-32 expression by fibroblast-like synoviocytes in rheumatoid arthritis. Arthritis Res Ther 2010, 12:R135.

28. Shoda H, Fujio K, Yamaguchi Y, Okamoto A, Sawada T, Kochi Y, Yamamoto K: Interactions between IL-32 and tumor necrosis factor alpha contribute to the exacerbation of immune-inflammatory diseases. Arthritis Res Ther 2006, 8:R166.

29. Jeong HJ, Shin SY, Oh HA, Kim MH, Cho JS, Kim HM: IL-32 up-regulation is associated with inflammatory cytokine production in allergic rhinitis. J Pathol 2011, 224:553-563.

30. Yagi $Y$, Andoh A, Imaeda H, Aomatsu T, Ohsaki R, Inatomi O, Bamba S, Tsujikawa T, Shimizu T, Fujiyama Y: Interleukin-32alpha expression in human colonic subepithelial myofibroblasts. Int J Mol Med 2011, 27:263-268.

31. Mun SH, Kim JW, Nah SS, Ko NY, Lee JH, Kim JD, Kim do K, Kim HS, Choi JD, Kim SH, Lee CK, Park SH, Kim BK, Kim HS, Kim YM, Choi WS: Tumor necrosis factor alpha-induced interleukin-32 is positively regulated via the Syk/protein kinase Cdelta/JNK pathway in rheumatoid synovial fibroblasts. Arthritis Rheum 2009, 60:678-685.

\section{doi:10.1186/ar4089}

Cite this article as: Moon et al:: IL-32 and IL-17 interact and have the potential to aggravate osteoclastogenesis in rheumatoid arthritis. Arthritis Research \& Therapy 2012 14:R246. 